\title{
Bio fabrication of gold nanoparticles and their combination with chemotherapy and ultrasound for effective treatment of tumors
}

\author{
Mingmei Li
}

Department of Ultrasound Medicine, Xintai People's Hospital.

\begin{abstract}
Background and Objective: Chemotherapy is one of the important medical option for the cancer therapy, but still new combination of therapeutic treatment methods are needed to achieve efficient anti tumor activity. In this work, Pueraria lobata leaf extract was utilized as a bio reductant for the fabrication of the Gold nanoparticles (Au-NPs) by an ecological approach without using any harmful chemical. This work also evaluates whether the combined effect of chemotherapy along with Au-NPs and Ultrasound (US) is effective over the tumors or not.

Materials and methods: About $10 \mathrm{~mL}$ of plant extract was added to $1 \mathrm{mM}$ of HAuCl4 solution $(20.0 \mathrm{~mL})$ and $\mathrm{pH}$ was maintained at 12 and allowed for stirring for half an hour. The change in solution color from yellow to purple signified the Au-NPs formation.

Results: XRD results and TEM images confirmed the formation of crystalline Au-NPs with sizes ranging from 23-30 nm. Further, biological studies revealed that the combination of Au-NPs and US along with chemotherapy improved the impact of anti-cancer drug.

Conclusion: In conclusion, Pueraria lobata leaf extract mediated synthesis of Au-NPs by an eco-friendly approach was reported in this work. The plant biomolecules of the extract were involved in the reduction and capping of the Au-NPs formed. The major conclusion is that addition of Au-NPs with chemotherapy and ultrasound has shown more effective anti-tumor activity.

Keywords: Au-NPs; chemotherapy; ultrasound; plant bio-constituents.

DOI: https://doi.org/10.4314/ahs.v20i2.35

Cite as: Li M. Bio fabrication of gold nanoparticles and their combination with chemotherapy and ultrasound for effective treatment of tumors. Afri Health Sci. 2020; 20(2): 815-821. https:// doi.org/10.4314/abs.v20i2.35
\end{abstract}

\section{Introduction}

Fabrication of gold nanoparticles (Au-NPs) has gained significant interest from researchers because of their very good thermal and chemical, along with some exceptional features such as catalytic, electronic and optical properties. Au-NPs exhibit great benefits in biological applications when compared to other NPs because of their no-toxic nature and very good cytocompatibility, resulting their wide scope of applications in drug delivery, tumor therapy, biosensors and bio imaging ${ }^{1-4}$.

Various physical and chemical approaches have been reported for production of Au-NPs, which possess several disadvantages such as non-eco-friendly nature and

\section{Corresponding author: \\ Mingmei Li, \\ No. 1329 Xinfu Road, Xintai 271200, \\ Shandong, PR China, \\ Email: lmingmei@yahoo.com}

high cost because of the involvement of toxic reducing agents. On the other hand, the usage of natural plant biomolecules, fungi and microbes for the preparation of nanomaterials resulting in an environmental friendly, cost effective and non-hazardous approach ${ }^{5}$.

In the green synthesis approaches, some biomolecules such as diastase ${ }^{6}$, tyrosine ${ }^{7}$, casein ${ }^{8}$, silk sericin ${ }^{9}$ plant extracts like Abelmoschusesculentus ${ }^{10}$, Azadirachtaindica ${ }^{11}$, Maducalongifolia ${ }^{12}$, Citrus sinensis ${ }^{13}$ and Hibiscus rosasinensis ${ }^{14}$ have been reported to be used, as they acts as reducing and capping agents for synthesis of various nanomaterials. Although several green methods have been reported for the fabrication of Au-NPs, still there is a requirement for the evolution of new techniques for the Au-NPs fabrication for their potential biological applications.

On the other hand, Ultrasound (US) and US contrast agents added with chemotherapy is a method that enhances the permeability of the cell membrane at the focused site and has specific advantages such as low invasiveness, low toxicity, repeated applicability and

(C) 2020 Li M. Licensee African Health Sciences. This is an Open Access article distributed under the terms of the Creative commons Attribution License (https://creativecommons.org/licenses/BY/4.0), which permits unrestricted use, distribution, and reproduction in any medium, provided the original work is properly cited. 
high tissue selectivity ${ }^{15-16}$. One of the major popular anti-cancer drugs, CDDP (Cis-Diamminedichloroplatinum (II)) is mostly utilized for treating various kinds of solid tumors ${ }^{17-19}$. The CDDP effective delivery into tumors in LNs may be greatly expected to improve its chemotherapeutic activity.

The objective of the current research is to biosynthesize $\mathrm{Au}-\mathrm{NPs}$ via a bio friendly approach and to evaluate the antitumor efficiency of combining Au-NPs and US along with CDDP. We determined that Au-NPs and US combined with CDDP is efficient in producing a prominent anti-tumor activity.

\section{Materials and methods \\ Materials}

Chloroauric acid $(\mathrm{HAuCl} 143 \mathrm{H} 2 \mathrm{O})$, Sodium hydroxide $(\mathrm{NaOH})$ and other chemicals were purchased from sigma aldrich chemicals, shanghai. The leaves of pueraria lobata were collected from Shenlongjia forest area, china. Milli-Q water was used for all experiments.

\section{Fabrication of $A u-N P s$}

Initially the obtained leaves of pueraria lobata were collected and dried under sunlight. Later, $4 \mathrm{~g}$ of above dried powder is added to $100 \mathrm{ml}$ of water and boiled for $30 \mathrm{~min}$ and the mixture was filtered using cellulose nitrate membrane filter paper. The filtrate was used for fabrication of Au-NPs. About $10 \mathrm{~mL}$ of plant extract was added to $1 \mathrm{mM}$ of $\mathrm{HAuCl} 4$ solution $(20.0 \mathrm{~mL})$ and $\mathrm{pH}$ was maintained at 12 and allowed for stirring for half an hour. The change in solution color from yellow to purple represented the Au-NPs formation.

\section{Cell preparation}

GFP/KM-Luc cells, expressing stably a fusion of green fluorescent and luciferase protein genes, were formed using transfection of fatal fibrous histiocytoma such as the (N/MRL-1) cells ${ }^{20}$ along with pEGFPLuc with the help of lipofectin transfection reagent. N/MRL-1 cells were a sarcoma cell line determined from the spleens of MpTn-gld/gld/MRL rats ${ }^{21}$. GFP/KM-Luc cells were stored in the medium, Dulbecco's modified Eagle added with fetal bovine serum of $10 \%$, geneticin $(0.5 \mathrm{mg} /$ $\mathrm{mL}, \mathrm{G} 418$ sulfate) and L-glutamine-penicillin-streptomycin of $1 \%$. The cells are incubated at a temperature of $37^{\circ} \mathrm{c}$ in a solution of carbon dioxide $5 \%$ and air $95 \%$ until achieving $80 \%$ confluence.

\section{In vitro US treatment}

GFP/KM-Luc cells are trypsinized using the solution of EDTA-trypsin (EDTA $0.02 \%$ and trypsin 0.25\%) and dissolved at a $4.0 \times 104$ cells $/ \mathrm{mL}$ concentration in the same medium utilized in the cell formation. Later, samples of $500-\mu \mathrm{L}$ were aliquoted in 48 -well plates and then incubated at a temperature of $37^{\circ} \mathrm{c}$ in the solution of carbon dioxide $5 \%$ and air $95 \%$. After $24 \mathrm{~h}$, the medium in every well plate was extracted and suspended in a $450 \mu \mathrm{L}$ of similar medium, which was utilized for the preparation of cell. Moreover, PBS of $50 \mu \mathrm{L}$ was added to the US control group; PBS and Au-NPs of $25 \mu \mathrm{L}$ each was added to the Au-NPs + US group; and for the $\mathrm{CDDP}+\mathrm{Au}-\mathrm{NPs}+\mathrm{CDDP}$ groups, CDDP of $25 \mu \mathrm{L}$ in PBS and Au-NPs of $25 \mu \mathrm{L}$ were added, maintaining the overall CDDP concentration as 250 or $50,10,1 \mu \mathrm{M}$. In every group, the samples were placed $100 \mathrm{~mm}$ overhead a $30 \mathrm{~mm}$ diameter US transducer dissolved in the tap water (degassed followed by heating to a temperature of $38^{\circ} \mathrm{c}$ ) and later may be exposed to US $(1.0,0.5,0.1$ $\mathrm{W} / \mathrm{cm} 2 ; 60 \mathrm{~s}$ ) or may not be exposed. After exposing to US, the samples are incubated at a temperature of $37^{\circ} \mathrm{c}$ in a solution of carbon dioxide $5 \%$ and air $95 \%$. After $24 \mathrm{~h}$, we determined the cell viability with the help of a MTT assay (3-(4,5-dimethylthiazol-2-yl)-2,5-diphenyltetrazolium bromide) as demonstrated earlier ${ }^{22}$. The samples that were not exposed to CDDP, Au-NPs and US were utilized as the controls. Six samples were used to perform every experiment. For every experiment, the results achieved by the treated samples were made to be divided by the average of the control samples for obtainig a normalized cell viability factor.

\section{Characterization}

UV-visible spectrophotometer (Thermo Scientific) was used to obtain the optical absorbance spectrum of the Au-NPs at a wavelength from 400 to $700 \mathrm{~nm}$. A Rigakumini Flex 11, XRD instrument was used to know the crystalline nature of $\mathrm{Au}-\mathrm{NPs}$ with $\mathrm{Cu} \mathrm{K} \alpha(\lambda=1.5406$ A), $15 \mathrm{~mA}$ current at an operating voltage of $30 \mathrm{kV}$. Tecnai G2 spirit BioTWIN, TEM instrument was utilized to analyse the size and shape of Au-NPs. Sample for TEM analysis was prepared by dispersing the purified centrifuged product in water under ultrasonication conditions. The as prepared dispersion was loaded and dried on a copper grid and allowed for evaporation and then visualized under TEM microscope. A Perkin Elmer FTIR instrument was used to know the surface capping of Au-NPs with plant polyphenols and the measurements were carried out at $400 \mathrm{~cm}-1$ to $4000 \mathrm{~cm}-1$ wavelength range.

\section{Statistical analysis}

All the statistical information provided were represent- 
ed as the mean \pm standard deviation (SD). Data was conducted with ANOVA. To determine the source of significant variations we utilized Dunnett range posthoc comparisons, wherever applicable. The value of $\mathrm{P}$ less than 0.05 was statistically considered as significant.

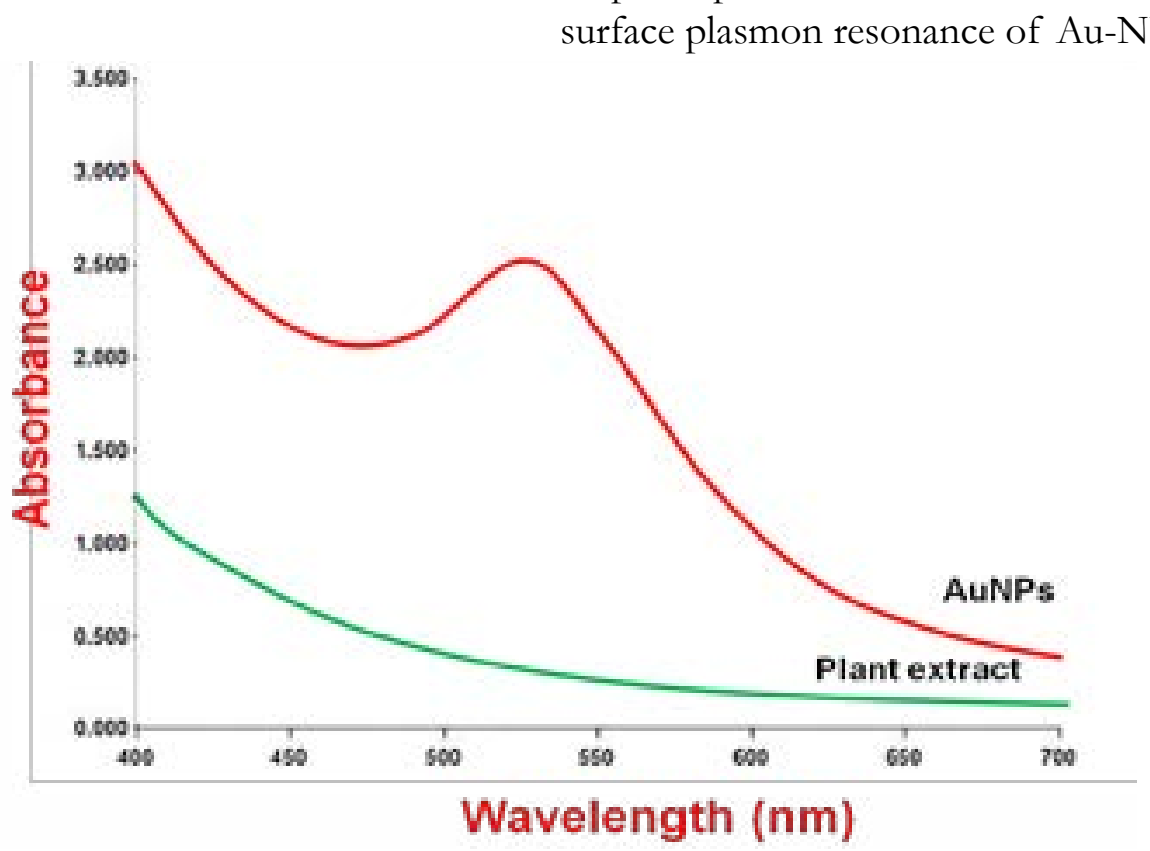

Fig.1. UV-Vis absorption spectrum of plant extract and Au-NPs

\section{Results and discussion}

From the UV-Vis spectral analysis (shown in Fig.1), it is found that no optical absorption peak is detected for plant extract. Whereas absorption band is observed at $529 \mathrm{~nm}$ indicated the generation of Au-NPs. This absorption peak of Au-NPs is due to phenomenon of surface plasmon resonance of Au-NPs.
From the Fig. 2, it is found that XRD pattern of $\mathrm{Au}-$ NPs exhibited $2 \theta$ values at $77.300^{\circ}, 64.400^{\circ}, 43.825^{\circ}$, $37.875^{\circ}$, with corresponding crystal planes at (311), (220), (200), and (111) respectively. The XRD pattern agrees with face cantered cubic structure of Au-NPs. The obtained XRD pattern is similar with the AuNPs prepared by using green reductants such as diastase 14 . Fig. 3. shows the TEM microscopic images of the formed Au-NPs. From the TEM images, it is observed that the formed Au-NPs were in hexagonal shape and the particle size ranges form 23-30 nm. Fig.4 showed the EDS spectrum of Au-NPs, which confirmed the presence of dominant Au elemental signals representing the preparation of Au-NPs. On the other hand, the presence of other elemental peaks may arise from plant extract bio constituents.

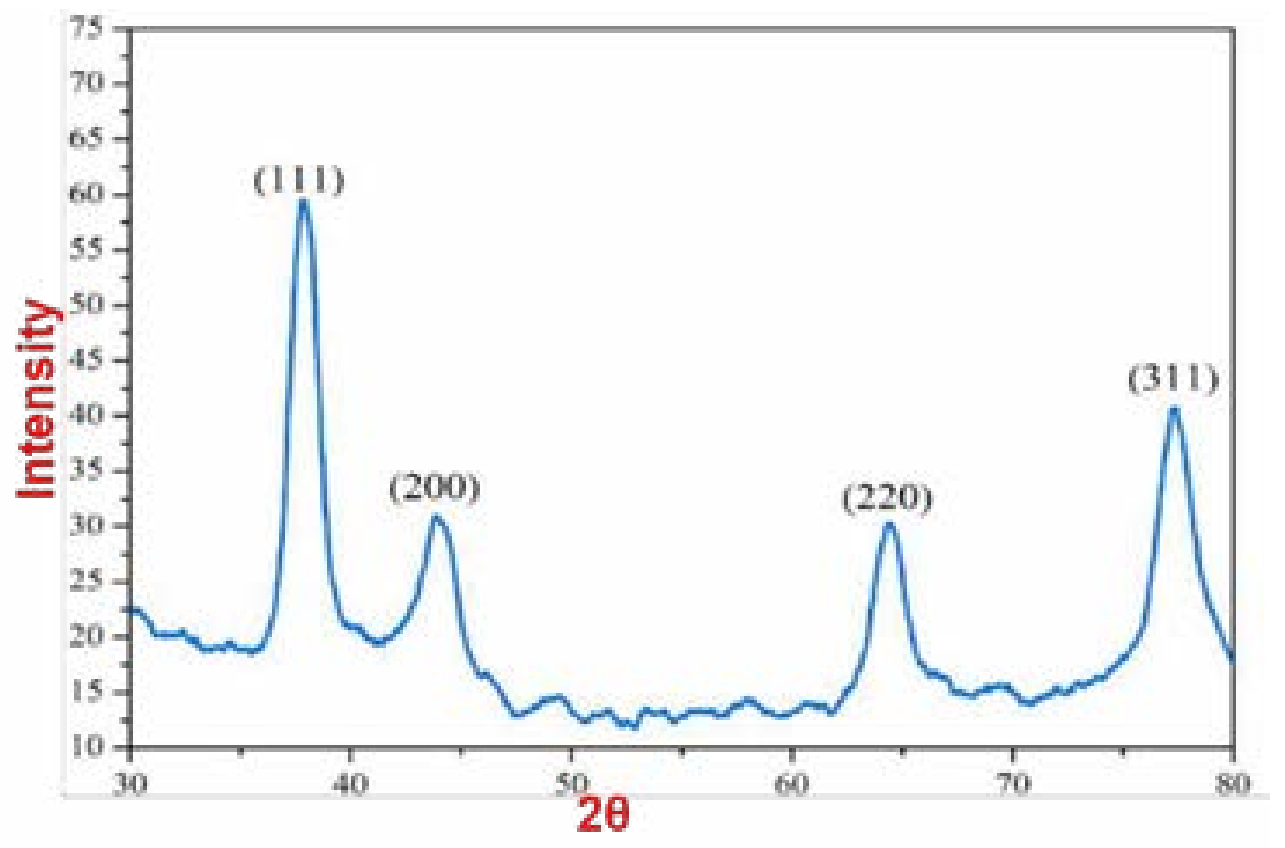

Fig.2. Au-NPs XRD pattern 


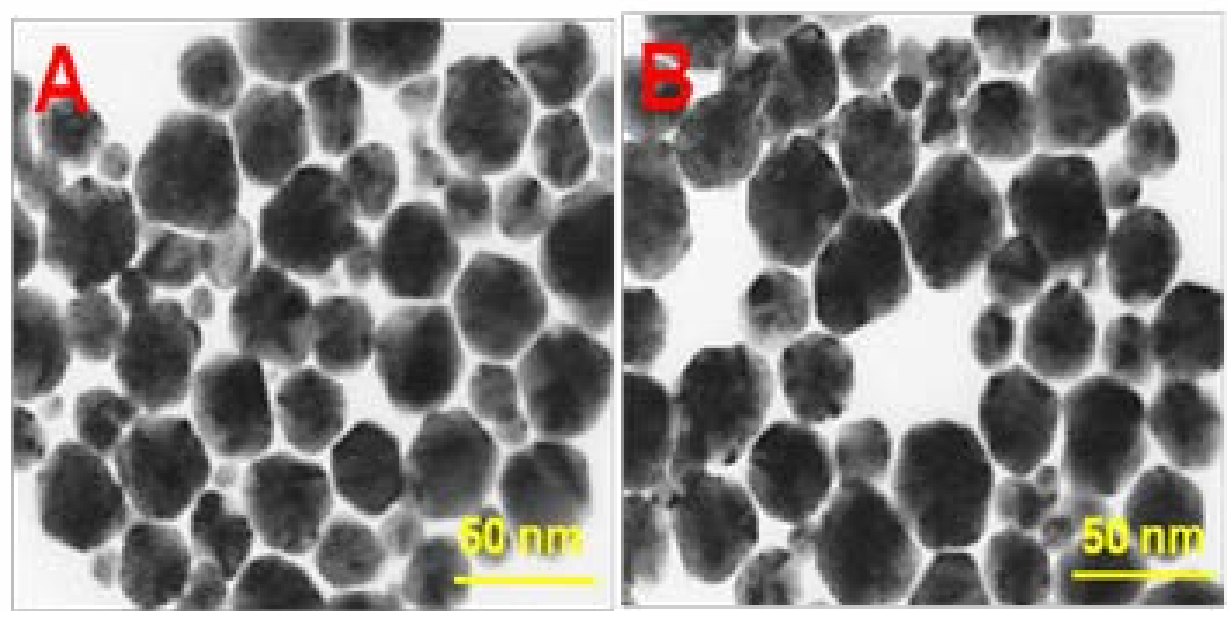

Fig.3. TEM images of prepared Au-NPs

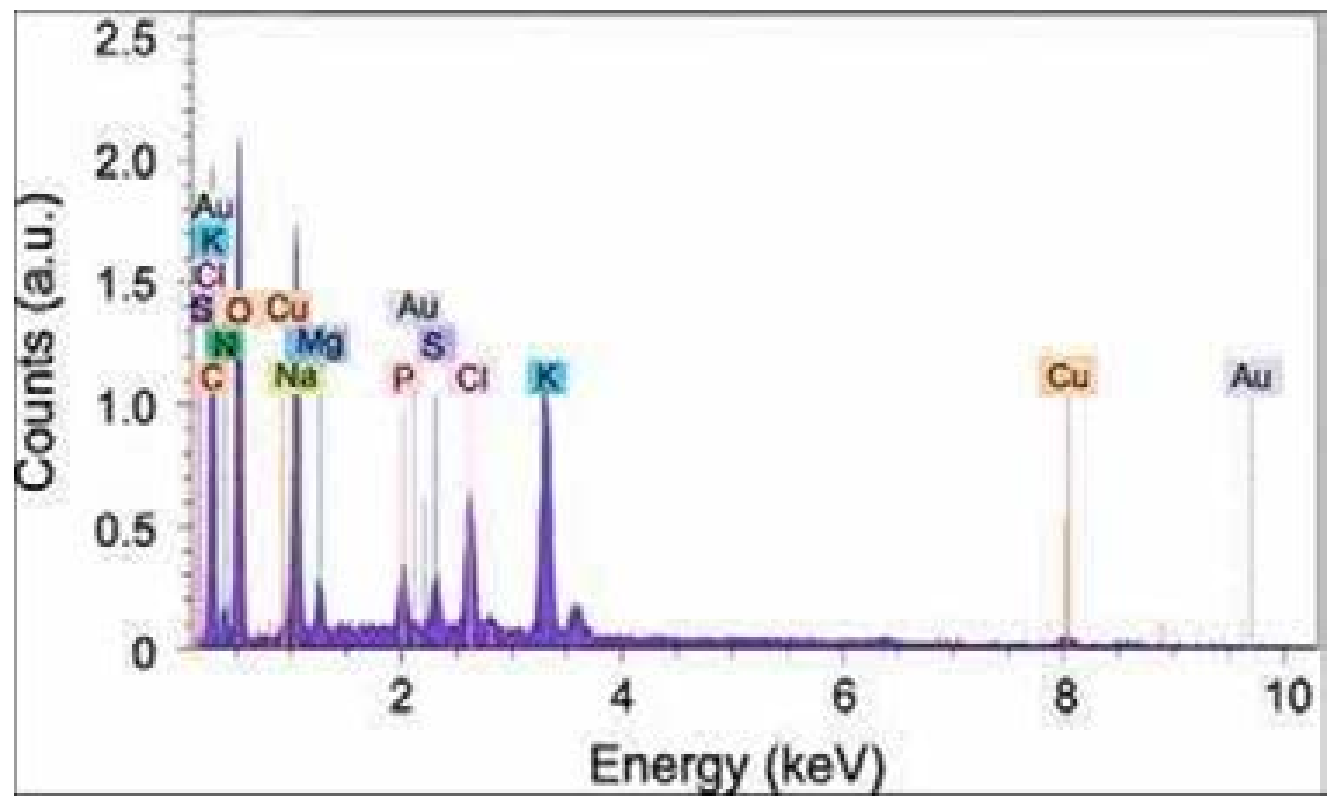

Fig.4. EDS spectrum of Au-NPs

Fig.5 represented the FTIR spectrum of plant extract and Au-NPs prepared. It is exhibited from FTIR spectrum that the $-\mathrm{OH}$ and aldehyde functionalities are adsorbed on the surface of Au-NPs while the reduction process continued. It is noted that the vibrational bands of alcoholic groups at $1255.00 \mathrm{~cm}^{-}{ }^{1}$, and phenolic functionalities of plant extract and -CHO groups at $1628.72 \mathrm{~cm}^{-1}$ and $542.85 \mathrm{~cm}^{-1}$ are considerably weaker in Au-NPs when compared to plant extract. It is also known from FTIR spectrum that there is a blue shift in bands of Au-NPs spectrum, signifying the change in the chemical environment of plant bio-constituents, indicating that $-\mathrm{OH}$, alcoholic and - $\mathrm{CHO}$ functionalities of plant extract are involved in capping of Au-NPs formed. Also, the presence of similar functionalities of green reagent used for reduction on the surface of prepared AuNPs further indicated the capping of plant extract polyphenols onto AuNPs ${ }^{14-18}$. 


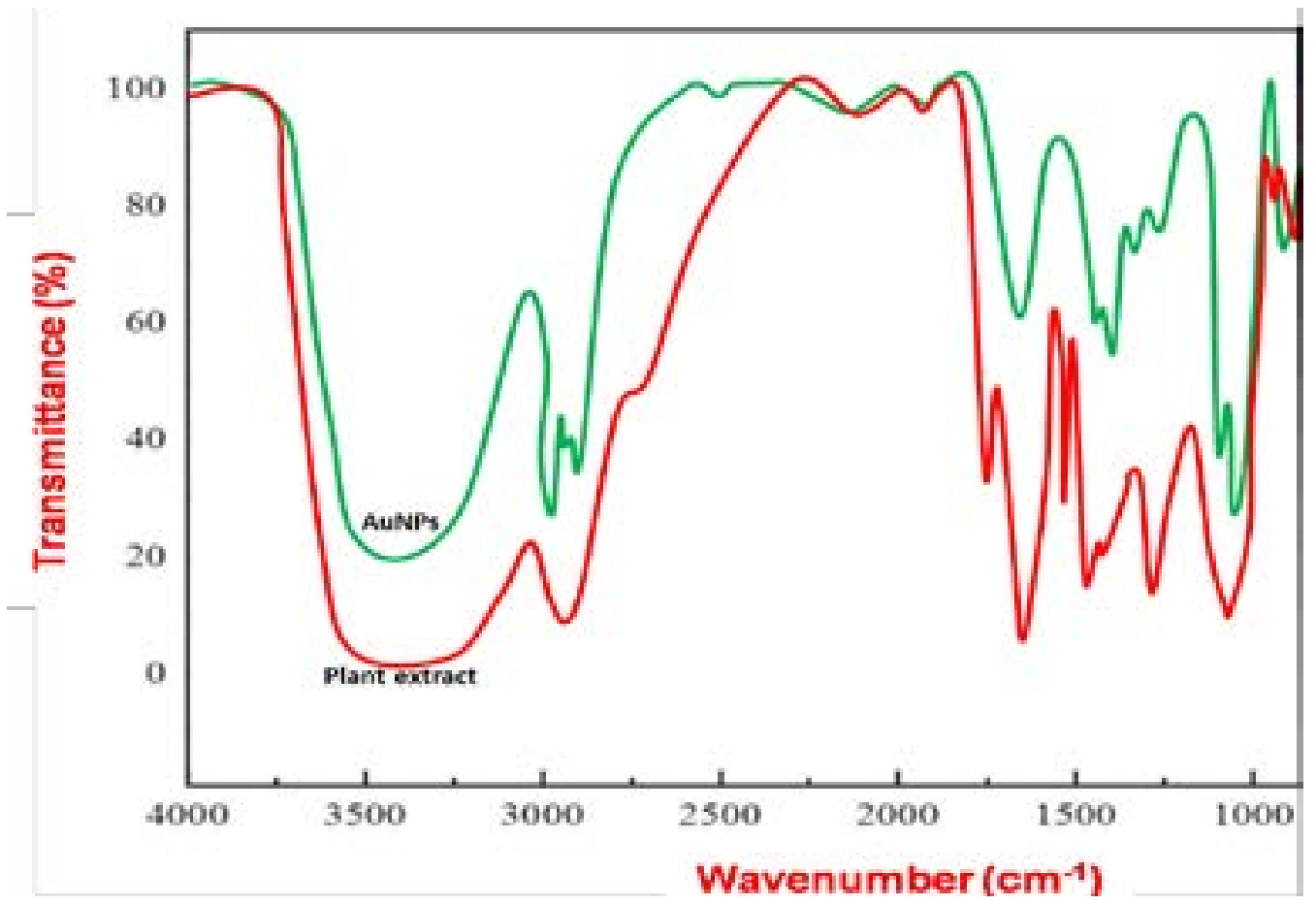

Fig. 5 FTIR spectrum of plant extract and Au-NPs prepared

\section{In vitro US treatment}

To determine whether a CDDP anti-tumor effect was enhanced by adding Au-NPs and US, each experimental group's cell viability was recorded using the MT'T assay (Fig. 6). Evaluation of the results for the US + Au-NPs + CDDP $(1 \mu \mathrm{M})$, Au-NPs + US and US control groups exposed an unique approach towards a normalized cell viability decrease depending on the intensity of US. A statistically substantial variance in normalized cell viability in-between the Au-NPs + CDDP and US + AuNPs + CDDP was noticed only when the concentration of CDDP used was at $10 \mu \mathrm{M}$ and the intensity of US was $1.0 \mathrm{~W} / \mathrm{cm}^{2}$ (where $\mathrm{p}$ less than 0.05) (Fig. 7). Au-NPs along with US did not considerably improve the anti-tumor activity of high $(250$ and $50 \mu \mathrm{M})$ or low $(1 \mu \mathrm{M})$ levels of CDDP injected.

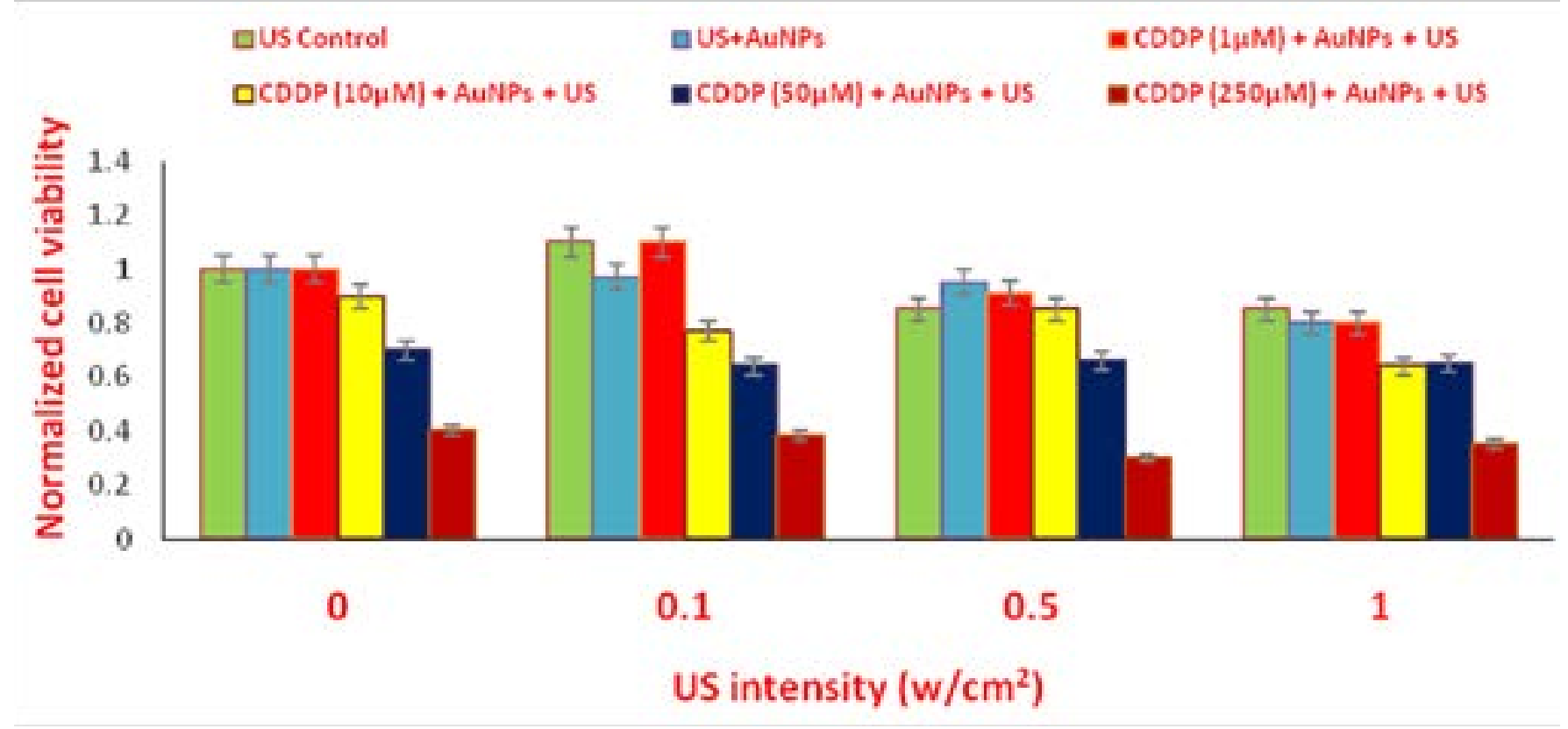

Fig. 6. Ultra sound intensity-dependent decrease in normalized cell viability of the US control, US +Au-NPs and CDDP+ Au-NPs (where CDDP is 1, 10, 50, 250 micro meter 


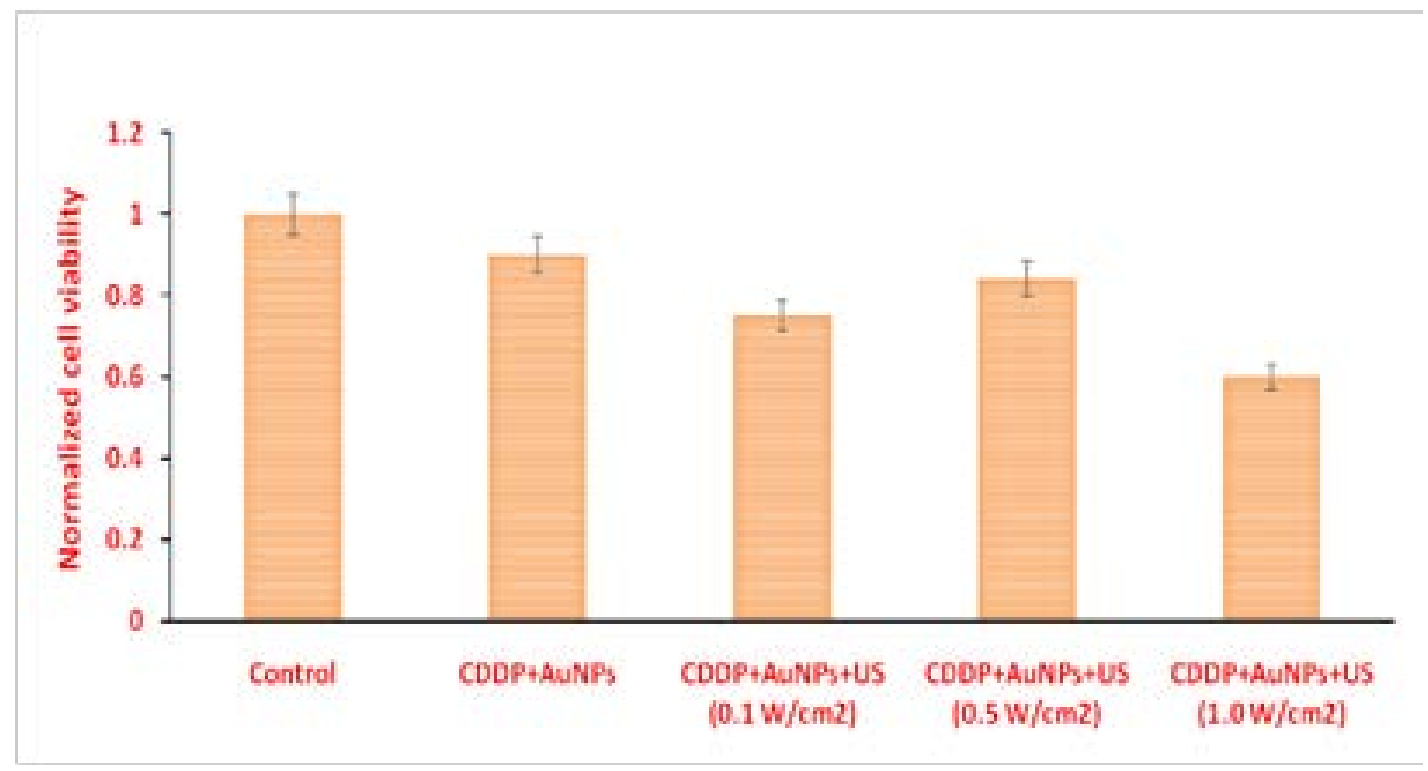

Fig.7. Normalized cell viability of the CDDP $+\mathrm{Au}-\mathrm{NPs}+\mathrm{US}$ (10 micro molar CDDP concentration), where the US intensity changes from $0.1,0.5$ and $1.0 \mathrm{~W} / \mathrm{cm}^{2}$

\section{Conclusion}

Pueraria lobata leaf extract mediated synthesis of $\mathrm{Au}-$ NPs by an eco-friendly approach was reported in this work. The plant biomolecules of extract are involved in reduction and capping of the formed Au-NPs, which was confirmed by FTIR spectroscopic results. XRD results and TEM images confirmed the formation of crystalline Au-NPs with size ranges form 23-30 nm. Further, biological studies revealed that the combination of Au-NPs and US along with chemotherapy improved the impact of anti-cancer drug. The major conclusion is that addition of Au-NPs with chemotherapy and ultrasound has shown more effective anti-tumor activity.

\section{Conflict of interest}

None declared.

\section{References}

1. Kotopoulis SA, Delalande M, Popa V, Mamaeva G, Dimcevski OH, Gilja M, Postema BT, Gjertsen E. McCormack, 2013. Sonoporationenhanced chemotherapy significantly reduces primary tumour burden in an orthotopic pancreatic cancer xenograft. Mol Imaging Biol., 16:53-62.

2. Watanabe YA, Aoi S, Horie N, Tomita S, Mori H, Morikawa Y, Matsumura G, Vassaux, T Kodama, 2008. Low-intensity ultrasound and microbubbles enhance the antitumor effect of cisplatin. Cancer Sci., 99:25252531.

3. Boulikas T, M. 2003. Vougiouka, Cisplatin and plat- inum drugs at the molecular level. Review. Oncol Rep., 10:1663-1682.

4. Rozencweig MR, Abele DD, von Hoff, FM Muggia, 1981. Cisplatin: Impact of a new anticancer agent on current therapeutic strategies. Anticancer Res., 1:199-204. 5. Spira AI, DS Ettinger, 2002. The use of chemotherapy in soft-tissue sarcomas. Oncologist., 7:348-359.

6. Li L, S Mori, M Sakamoto, S Takahashi, T Kodama, 2013. Mouse model of lymph node metastasis via afferent lymphatic vessels for development of imaging modalities. PLoS One., 8(2): e55797.

7. Hasegawa H, M Kohno, M Sasaki, A Inoue, MR Ito, M Terada, K Hieshima, H Maruyama, J Miyazaki, O Yoshie, M Nose, S Fujita, 2003. Antagonist of monocyte chemoattractant protein 1 ameliorates the initiation and progression of lupus nephritis and renal vasculitis in MRL/lpr mice. Arthritis Rheum., 48:2555-2566.

8. Kodama T, AG Doukas, MR Hamblin, 2003. Delivery of ribosome-inactivating protein toxin into cancer cells with shock waves. Cancer Lett., 189:69-75.

9. Alric CJ, Taleb GL, Duc C, Mandon C, Billotey AL, MeurHerland T, Brochard F, Vocanson M, Janier P, Perriat S, Roux O. Tillement, 2008. Gadolinium Chelate Coated Gold Nanoparticles As Contrast Agents for Both X-ray Computed Tomography and Magnetic Resonance Imaging. J. Am. Chem. Soc., 130:5908-5915. 10. Cai W, T Gao, H Hong, J Sun, 2008. Applications of gold nanoparticles in cancer nanotechnology. Nanotechnol. Sci. Appl., 1:17-32.

11. Ghosh PG, Han M De, CK Kim, VM. Rotello, 2008. Gold nanoparticles in delivery applications. Adv. Drug Deliv. Rev., 60:1307-1315.

African Health Sciences Vol 20 Issue 2, June, 2020 
12. Salata OV, 2004. Applications of nanoparticles in biology and medicine. J. Nanobiotechnol., 2:3-8.

13. Sireesh Babu M, M Badal Kumar, 2014. A review on 'Low-cost and eco-friendly green methods for graphene synthesis. International journal of nanoscience and technology., 3:2319-8796.

14. Sireesh Babu M, M Badal Kumar, R Shivendu, D Nandita, 2015. Diastase assisted green synthesis of size controllable gold nanoparticles. RSC Advances., 5:2672726733.

15. Sireesh Babu M, M Badal Kumar, A Kiran Kumar, 2017. Tyrosine assisted size controlled synthesis of silver nanoparticles and their catalytic and in-vitro cytotoxicity evaluation. Environmental Toxicology and Pharmacology., 51:23-29.

16. Sireesh Babu M, M Badal Kumar, V Raviraj, K Poliraju, T Sai Kumar, HA Kiran Kumar, 2014. Casein mediated green synthesis and decoration of reduced graphene oxide. Spectrochimica Acta Part A: Molecular and Biomolecular Spectroscopy., 126:227- 231.

17. Sireesh Babu M, S Jegatheeswaran, SY Serap, H Guobin, C Yurong, Junkuo Gao, Qingqing Ni, Juming Yao, 2018. Silk sericin induced fabrication of reduced graphene oxide and its in-vitro cytotoxicity, photothermal evaluation. Journal of Photochemistry \& Photobiology, B: Biology., 186:189-196.

18. Jayaseelan C, R Ramkumar, AA Rahuman, P Perumal, 2013. Green synthesis of gold nanoparticles using seed aqueous extract of Abelmoschus esculentus and its antifungal activity. Ind. Crops Prod., 45:423-429.

19. Shankar SS, A Rai, A Ahmad, M Sastry, 2004. Rapid synthesis of $\mathrm{Au}, \mathrm{Ag}$, and bimetallic Au Core-Ag shell nanoparticles using Neem (Azadirachta indica) leaf broth. J. Colloid Interface Sci., 275:496-502.

20. Fayaz AM, M Girilal, R Venkatesan, PT Kalaichelvan, 2011. Biosynthesis of anisotropic gold nanoparticles using Maduca longifolia extract and their potential in infrared absorption. Colloids Surf. B. Biointerfaces., 88:287-291.

21. Shankar SS, A Rai, A Ahmad, M Sastry, 2004. Rapid synthesis of $\mathrm{Au}, \mathrm{Ag}$, and bimetallic Au Core-Ag shell nanoparticles using Neem (Azadirachta indica) leaf broth. J. Colloid Interface Sci., 275:496-502.

22. Philip D, 2010. Green synthesis of gold and gold nanoparticles using Hibiscus rosa sinensis. Phys. E Low Dimens. Syst. Nanostruct., 42:1417-1424. 